\title{
OPEN Clustering of cardio-metabolic risk factors and pre-diabetes among U.S. adolescents
}

\author{
Chibo Liu, Susu Wu \& Xiao Pan ${ }^{凶}$
}

Few studies have assessed the association between clustering of cardio-metabolic risk factors (CMRFs) and pre-diabetes in children or adolescents. We aimed to examine the association between clustering of CMRFs and pre-diabetes among U.S. adolescents. Data were available for 5,633 U.S. adolescents aged 12-19 years from the National Health and Nutrition Examination Surveys 1999-2014. Prediabetes was defined as impaired fasting glucose (IFG) (fasting plasma glucose 100-125 mg/dL), impaired glucose tolerance (IGT) (2-h plasma glucose 140-199 mg/dL) or elevated hemoglobin A1c (HbA1c) (HbA1c 5.7-6.4\%). The individual CMRFs considered in the present study were as follows: waist-to-height ratio, blood pressure, triglycerides, and high-density lipoprotein cholesterol. CMRFs were defined based on the modified National Cholesterol Education Program (NCEP) criteria or the modified International Diabetes Federation (IDF) criteria. Logistic regression analysis was used to examine the association between clustering of CMRFs and pre-diabetes with adjustment for potential covariates. Among 5633 adolescents, $11.4 \%$ had IFG, $4.7 \%$ had IGT, $4.5 \%$ had elevated $\mathrm{HbA1C}$ and $16.1 \%$ had pre-diabetes. Compared with adolescents with no CMRFs, the odds ratios (ORs) with $95 \%$ confidence intervals (Cls) for pre-diabetes across the clustering of CMRFs (i.e., 1, 2, 3, and 4) were 1.32 (1.03-1.68), 2.07 (1.55-2.76), 2.52 (1.69-3.76), and 5.41 (3.14-9.32), respectively, based on the modified NCEP criteria. The corresponding ORs with $95 \%$ Cls were 1.16 (0.89-1.51), 1.78 (1.35-2.36), 3.07 (1.89-4.98) and 12.20 (3.93-37.89), respectively, based on the modified IDF criteria. The present study suggests that the clustering of CMRFs is associated with increased pre-diabetes among U.S. adolescents. It might be necessary for effective strategies and measures targeting adolescents with clustering of CMRFs, including those with less than 3 risk factors.

Diabetes has been a serious public health issue among the U.S. population. The economic burden associated with diabetes-related problems in the U.S. exceeded 322 billion dollars in $2012^{1}$. In addition, according to the Centers for Disease Control and Prevention in the U.S., there were approximately 84.1 million adults with prediabetes in $2015^{2}$. The prevalence of pre-diabetes among U.S. adolescents increased significantly from 1999 to $2014(1.9 \% \text { to } 5.0 \%)^{3}$. Fortunately, with early intervention, pre-diabetes can be reversible, especially in children and adolescents ${ }^{4}$. Thus, it is important for early intervention to build healthy habits that might prevent them from having diabetes and diabetes-related chronic diseases in adulthood.

With the epidemic of pediatric obesity, multiple cardio-metabolic risk factors (CMRFs) have been clustered in children and adolescents. Metabolic syndrome (MetS) is diagnosed when three or more CMRFs appear. However, the clinical utility of MetS in the pediatric population has been questioned ${ }^{5}$. Major concerns include a lack of uniformed criteria with many different pediatric MetS definitions, the instability of the dichotomous diagnosis, and no uniformed treatment for MetS rather than weight loss. Furthermore, although MetS in adults has been shown to be a well predictor for development of type 2 diabetes mellitus ${ }^{6}$, its values in children and adolescents are still challenging. Previous studies reported that MetS had low sensitivity in identifying adolescents with pre-diabetes ${ }^{7}$. Of note, the Bogalusa Heart Study showed significant increase in severity of atherosclerotic lesions associated with the increased clustering of CMRFs ${ }^{8}$. Thus, the American Academy of Pediatrics in 2017 recommends that it is better to focus on the concept of clustering of CMRFs rather than the definition of MetS in children or adolescents ${ }^{9}$. Identifying children or adolescents with multiple CMRFs may help target focused interventions on those with the highest risk for cardio-metabolic disease ${ }^{9}$. However, it is still unclear whether the risk of pre-diabetes in adolescents also increases with the clustering of CMRFs. 
Therefore, in the present study, we aimed to assess the association between clustering of CMRFs and risk of pre-diabetes among adolescents using data from the National Health and Nutrition Examination Surveys (NHANES) 1999-2014.

\section{Methods and materials}

Study population. The NHANES is a continuous, nationwide survey conducted by the National Center for Health Statistics (NCHS) of the Centers for Disease Control and Prevention. A stratified, multistage probability sampling design was used to obtain a representative sample of the civilian non-institutionalized resident population in the U.S. It has been conducted in 2-year cycles since 1999 with the goal of monitoring the health and nutritional status of U.S. children, adolescents, and adults. Participants were invited to complete household interviews, followed by physical examinations and laboratory tests. More detailed information about the NHANES is available online ${ }^{10}$. Written informed consent was obtained from adolescents aged 18 years or older. For adolescents aged 12 to 17 years, the content was signed by both adolescents and their parents/guardians. The survey protocol was approved by the NCHS Research Ethics Review Board. The NHANES data are publicly available without personal identifiable information and exempt under the ethical board review of the corresponding author's institution. All methods were carried out in accordance with relevant guidelines and regulations.

This study was limited to adolescents aged 12-19 years. To produce reliable estimates, we combined the 1999-2014 NHANES data for analysis ${ }^{11}$. We used multiple imputation method to fill in data if participants with missing information on all variables of interests. After the exclusion of participants with diagnosed (fasting plasma glucose $(\mathrm{FPG}) \geq 126 \mathrm{mg} / \mathrm{dL}$, or 2 -h glucose $\geq 200 \mathrm{mg} / \mathrm{dL}$, or Hemoglobin Alc $(\mathrm{HbAlc}) \geq 6.5 \%)^{12}$ and undiagnosed diabetes (self-reported physician-diagnosis of diabetes) from the analysis, a total of 5,633 adolescents with normal glucose or pre-diabetes were finally included in this study.

Measurement of CMRFs. CMRFs considered in the present study were as follows: waist-to-height ratio (WHtR), blood pressure (BP), triglycerides (TG), and high-density lipoprotein cholesterol (HDL-C). The physical examinations and laboratory tests were conducted at a mobile examination center. Waist circumference (WC) was measured to the nearest $0.1 \mathrm{~cm}$ using a standard tape. The tape was extended around the waist at the level of the uppermost lateral border of the ilium, and the reading was recorded at minimal respiration ${ }^{13}$. WHtR was calculated as WC divided by height. BP was measured by physicians using a mercury sphygmomanometer with the participants sitting straight and arm at the level of heart after at least 5 min rest. The average of the last two readings was used when three measures were taken ${ }^{13}$. Details of blood collecting and processing in NHANES are available elsewhere ${ }^{13}$. In brief, blood sample was drawn by trained nurses from adolescents who had completed at least an 8 -h fast. Blood specimens were placed in $-70^{\circ} \mathrm{C}$ Freezer until testing. TG and HDL-C were measured enzymatically.

Measurement of glucose and hemoglobin A1c. FPG and HbAlc were measured using the blood sample drawn in the morning after at least an 8-h fast. Then, an oral glucose tolerance test was administered. Participants were asked to drink $75 \mathrm{~g}$ glucose and two hours later, a second blood sample was drawn to obtain 2-h PG. FPG and 2-h PG were measured using hexokinase enzymatic methods and HbAlc was measured using highperformance liquid chromatography methods ${ }^{13}$. As glucose measurement methods have changed over time, to ensure comparability with earlier NHANES, we calibrated glucose data using regression equations provided in the NHANES data documentation ${ }^{14,15}$.

Potential confounders. Covariates adjusted in this study include sex, age, race/ethnicity (Hispanic, NonHispanic white, Non-Hispanic black, and others), and survey year, which were collected using the questionnaire.

Definitions of pre-diabetes. Impaired fasting glucose (IFG) was defined as having FPG 100-125 mg/ $\mathrm{dL}^{12}$. Impaired glucose tolerance (IGT) was defined as having 2-h PG $140-199 \mathrm{mg} / \mathrm{dL}^{12}$. Elevated HbA1c was defined as having HbA1c 5.7-6.4\% ${ }^{12}$. According to the American Diabetes Association (ADA) criteria, individuals with IFG, IGT, or elevated HbA1c and without diagnosed or undiagnosed diabetes were classified as having pre-diabetes ${ }^{12}$.

Definitions of CMRFs. Since there is no universal definition for CMRFs in children and adolescents, we used two international MetS criteria to determine whether participants had any of the four CMRFs (i.e. central obesity, elevated TG, low HDL-C, and elevated BP) that are components of the MetS. The two international MetS criteria included the modified International Diabetes Federation (IDF) criteria ${ }^{16}$ and the modified National Cholesterol Education Program (NCEP) criteria ${ }^{17}$. For the modified IDF definition, central obesity was defined as WHtR $\geq 0.50$; elevated $\mathrm{BP}$ was defined as systolic $\mathrm{BP} \geq 130 \mathrm{mmHg}$ or diastolic $\mathrm{BP} \geq 85 \mathrm{mmHg}$; elevated TG was defined as TG $\geq 150 \mathrm{mg} / \mathrm{dL}$; and low HDL-C was defined as $\mathrm{HDL}-\mathrm{C}<40 \mathrm{mg} / \mathrm{dL}$ for those aged $<16$ years or HDL-C $<40 \mathrm{mg} / \mathrm{dL}$ in males and $<50 \mathrm{mg} / \mathrm{dL}$ in females for those aged $\geq 16$ years $^{16}$. For the modified NCEP definition, central obesity was defined as $\mathrm{WHtR} \geq 0.50$; elevated $\mathrm{BP}$ was defined as systolic/diastolic $\mathrm{BP} \geq 90^{\text {th }}$ percentile; elevated TG was defined as TG $\geq 110 \mathrm{mg} / \mathrm{dL}$; and low HDL-C was defined as HDL-C $\leq 40 \mathrm{mg} / \mathrm{dL}^{17}$.

The clustering of CMRFs was defined as the sum of the four individual risk factors with each individual risk factor categorized as 0 vs. 1 . Thus, five categories were created, ranging from 0 to 4 .

Statistical analysis. Continuous variables were presented as means (standard errors [SE]), and the categorical variables were expressed as percentages. Differences in FPG, 2-h PG, and HbAlc across the five categories 


\begin{tabular}{|c|c|c|c|c|c|c|}
\hline & \multicolumn{5}{|c|}{ Clustering of cardio-metabolic risk factors } & \multirow[b]{2}{*}{$P$-value } \\
\hline & 0 & 1 & 2 & 3 & 4 & \\
\hline$N$ & 2540 & 1765 & 831 & 401 & 96 & \\
\hline \multicolumn{6}{|l|}{ Sex, \% } & $<0.0001$ \\
\hline Male & 50.7 & 46.1 & 53.5 & 63.6 & 86.5 & \\
\hline Female & 49.3 & 53.9 & 46.5 & 36.4 & 13.5 & \\
\hline \multicolumn{6}{|l|}{ Age (years), \% } & $<0.0001$ \\
\hline $12-15$ & 57.0 & 44.6 & 42.2 & 37.5 & 32.1 & \\
\hline $16-19$ & 43.0 & 55.4 & 57.8 & 62.5 & 67.9 & \\
\hline \multicolumn{6}{|l|}{ Race/ethnicity, \% } & $<0.0001$ \\
\hline Hispanic & 15.5 & 18.7 & 23.7 & 23.0 & 17.6 & \\
\hline Non-Hispanic white & 59.7 & 60.5 & 57.2 & 62.9 & 70.6 & \\
\hline Non-Hispanic black & 15.9 & 15.2 & 13.4 & 8.9 & 5.6 & \\
\hline Others & 8.9 & 5.6 & 5.6 & 5.2 & 6.2 & \\
\hline BMI, $\mathrm{kg} / \mathrm{m}^{2}$ & $20.2(0.1)$ & $24.6(0.2)$ & $27.1(0.3)$ & $30.7(0.5)$ & $33.0(0.6)$ & $<0.0001$ \\
\hline $\mathrm{WC}, \mathrm{cm}$ & $72.5(0.2)$ & $84.0(0.4)$ & $90.7(0.7)$ & $101.0(1.1)$ & $107.9(1.6)$ & $<0.0001$ \\
\hline WHtR & $0.438(0.001)$ & $0.505(0.003)$ & $0.544(0.004)$ & $0.599(0.006)$ & $0.616(0.010)$ & $<0.0001$ \\
\hline SBP, mmHg & $105.3(0.3)$ & $110.0(0.3)$ & $113.0(0.6)$ & $117.0(0.7)$ & $126.5(0.7)$ & $<0.0001$ \\
\hline $\mathrm{DBP}, \mathrm{mmHg}$ & $60.5(0.3)$ & $61.1(0.4)$ & $61.9(0.5)$ & $63.4(0.8)$ & $64.0(1.5)$ & $<0.0001$ \\
\hline $\mathrm{TG}, \mathrm{mg} / \mathrm{dL}$ & $63.3(0.7)$ & $82.4(1.4)$ & $117.3(3.2)$ & $157.4(5.6)$ & $182.1(8.7)$ & $<0.0001$ \\
\hline HDL-C, mg/dL & $56.9(0.3)$ & $51.1(0.4)$ & $44.4(0.5)$ & $38.3(0.5)$ & $33.7(0.6)$ & $<0.0001$ \\
\hline
\end{tabular}

Table 1. Characteristics of U.S. adolescents aged 12-19 years, NHANES 1999-2014. Continuous variables are expressed as mean (SE).

\begin{tabular}{|c|c|c|c|c|c|c|}
\hline & \multicolumn{5}{|c|}{ Clustering of cardio-metabolic risk factors } & \multirow[b]{2}{*}{$P$-value } \\
\hline & 0 & 1 & 2 & 3 & 4 & \\
\hline \multicolumn{7}{|l|}{ NCEP criteria } \\
\hline $\mathrm{FPG}, \mathrm{mg} / \mathrm{dL}$ & $90.7(0.2)$ & $91.3(0.2)$ & $92.1(0.3)$ & $93.0(0.5)$ & $95.6(0.8)$ & $<0.0001$ \\
\hline 2-h PG, mg/dL & $92.6(1.5)$ & $96.9(1.3)$ & $102.2(1.7)$ & $111.8(3.1)$ & $110.0(5.3)$ & $<0.0001$ \\
\hline $\mathrm{HbA}_{1 \mathrm{C}}, \mathrm{mg} / \mathrm{dL}$ & $5.12(0.01)$ & $5.14(0.01)$ & $5.15(0.01)$ & $5.19(0.02)$ & $5.20(0.05)$ & 0.0622 \\
\hline \multicolumn{7}{|l|}{ IDF criteria } \\
\hline $\mathrm{FPG}, \mathrm{mg} / \mathrm{dL}$ & $90.9(0.2)$ & $91.4(0.2)$ & $92.2(0.3)$ & $93.3(0.7)$ & $98.1(1.1)$ & $<0.0001$ \\
\hline 2-h PG, mg/dL & $93.6(1.4)$ & $98.5(1.2)$ & $103.2(2.0)$ & $114.8(4.2)$ & $106.0(13.9)$ & $<0.0001$ \\
\hline $\mathrm{HbA}_{1 \mathrm{C}}, \mathrm{mg} / \mathrm{dL}$ & $5.13(0.01)$ & $5.14(0.01)$ & $5.17(0.02)$ & $5.19(0.04)$ & $5.29(0.06)$ & 0.0220 \\
\hline
\end{tabular}

Table 2. Mean FPG, 2-h PG and $\mathrm{HbA}_{1 \mathrm{C}}$ levels according to clustering of cardio-metabolic risk factors. Adjusted for sex, age, race/ethnicity, and survey years.

of CMRFs were compared using covariance analysis adjusted for sex, age, race/ethnicity, and survey years. Chisquare test was used for comparison of categorical variables across the five categories of CMRFs. In addition, logistic regression models were used to estimate the odds ratios (ORs) with 95\% confidence intervals (CIs) of clustering of CMRFs associated with IFG, IGT, elevated HbAlc, and pre-diabetes, respectively, with adjustment for potential confounding factors. The NHANES uses a multistage sampling design, thus the sample design variables (strata, cluster and weights) were accounted for in the analyses for generalizability of the estimates. We performed all analyses using SAS version 9.3 (SAS, Cary, North Carolina, USA). Two-sided $P$ value $<0.05$ was considered to be statistically significant.

\section{Results}

Of the 5,633 adolescents included in this study, 11.4\% had IFG, 4.7\% had IGT, 4.5\% had elevated HbA1c, 13.0\% had both IFG and IGT, $14.8 \%$ had both IFG and elevated HbA1c, $6.2 \%$ had both IGT and elevated HbA1c, and $16.1 \%$ had pre-diabetes (either IFG, IGT, or elevated HbA1c). Table 1 shows the characteristics of U.S. adolescents aged 12-19 years according to the clustering of CMRFs (based on NCEP criteria). Significant differences in all characteristics were found across the five categories of CMRFs (all $P<0.0001$ ). In general, participants with clustering of CMRFs were more likely to be male, adolescents aged 16-19 years, Non-Hispanic white, to have abnormal anthropometric indices (BMI, WC, BP) and lipid profiles (TG and HDL-C) $($ all $P<0.0001)$.

Table 2 shows mean FPG, 2-h PG, and HbA1c levels according to the clustering of CMRFs. With the clustering of CMRFs, FPG, 2-h PG and HbA1c levels increased gradually, from $90.7 \mathrm{mg} / \mathrm{dL}$ to $95.6 \mathrm{mg} / \mathrm{dL}$, from $92.6 \mathrm{mg} / \mathrm{dL}$ to $110.0 \mathrm{mg} / \mathrm{dL}$ and from $5.12 \%$ to $5.20 \%$, respectively, based on the modified NCEP criteria; the 


\section{A. Based on NCEP criteria}

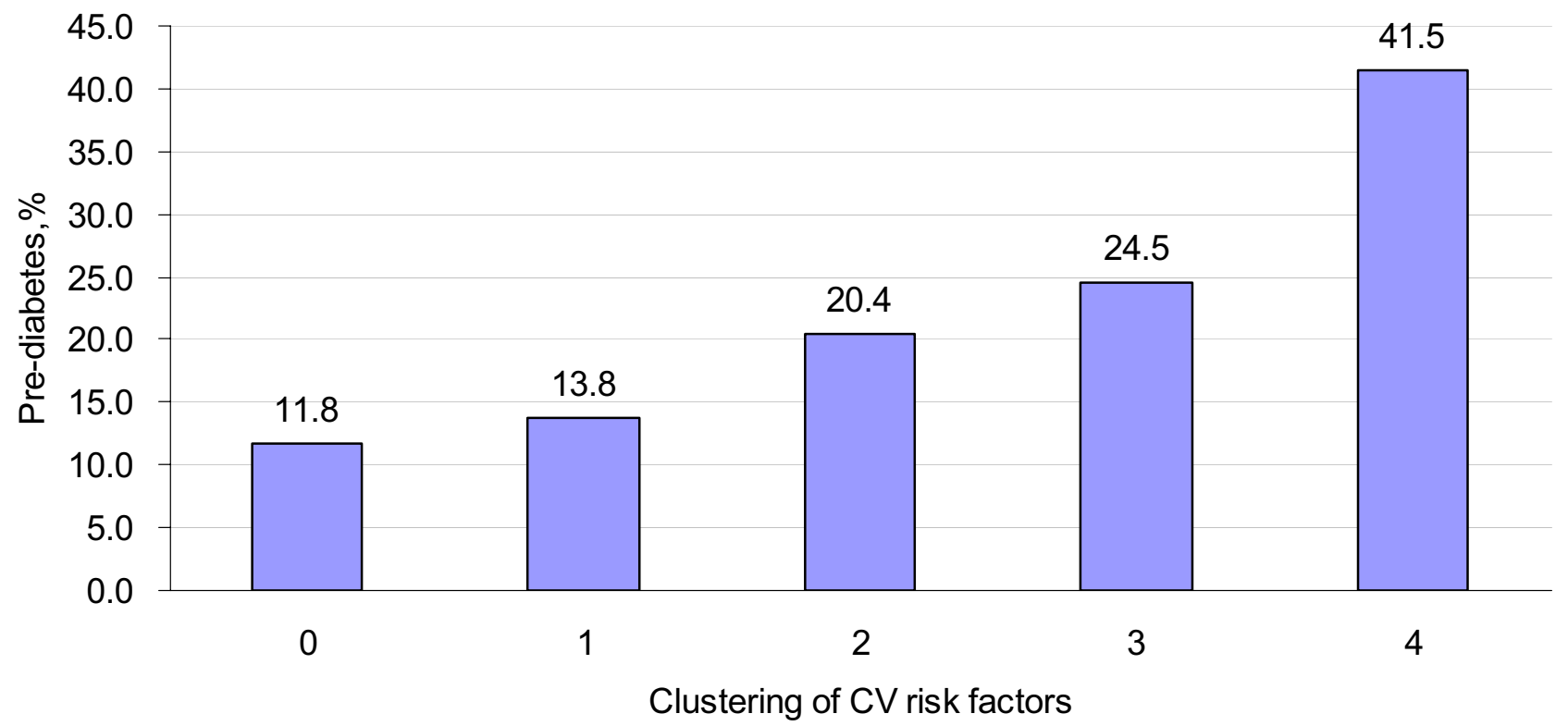

\section{B. Based on IDF criteria}

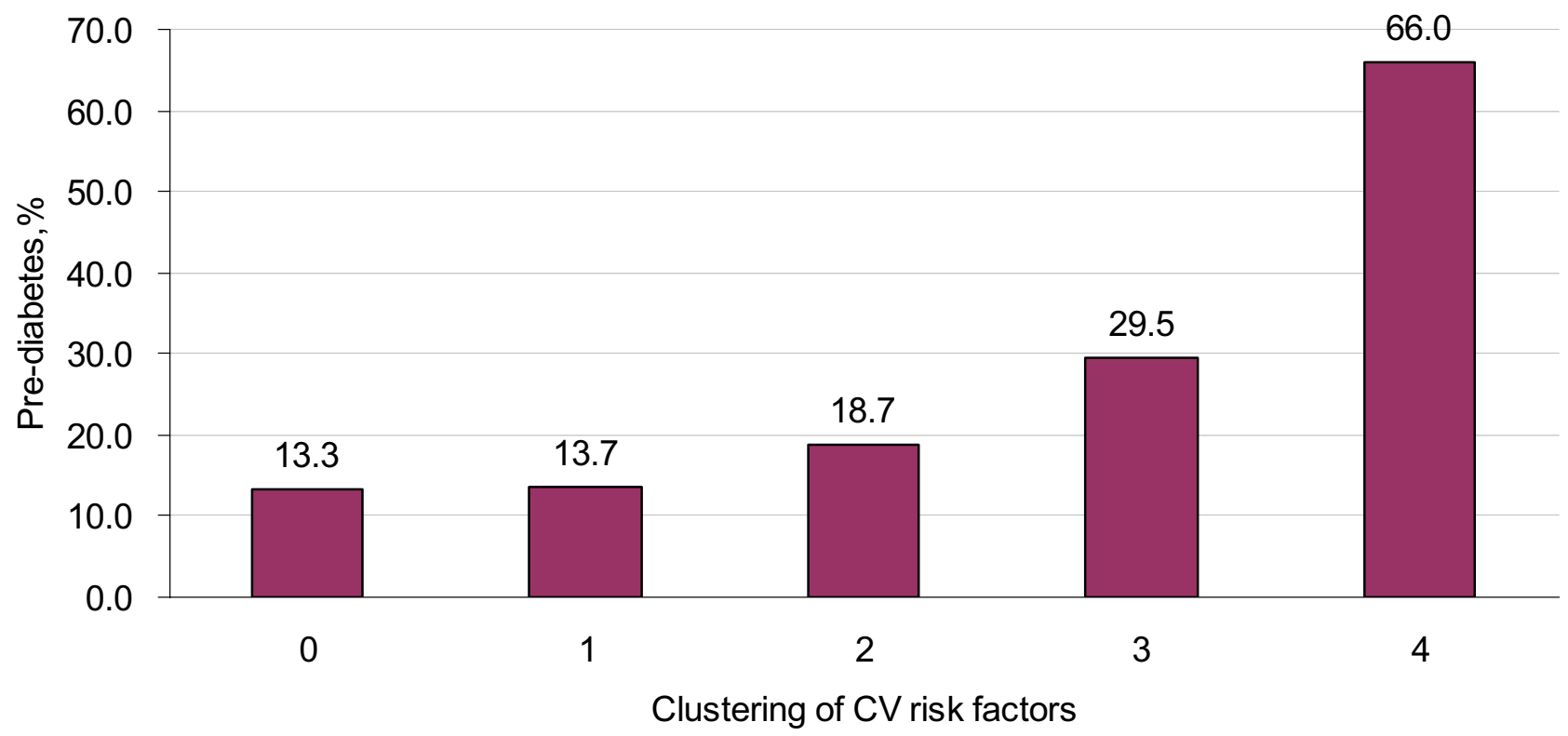

Figure 1. Prevalence of pre-diabetes according to clustering of cardio-metabolic risk factors based on (A) NCEP criteria and (B) IDF criteria.

prevalence of pre-diabetes also increased gradually with the clustering of CMRFs. For adolescents with $0,1,2$, 3 and 4 CMRFs (defined according to the modified NCEP criteria), the prevalence of pre-diabetes was $11.8 \%$, $13.8 \%, 20.4 \%, 24.5 \%, 41.5 \%$, respectively ( $P$ for trend $<0.001$, Fig. 1 A). Similar results were found according to the modified IDF criteria (Fig. 1B).

Logistic regression analyses adjusted for sex, age, race/ethnicity, and survey years showed that the risk of pre-diabetes tended to increase with the clustering of CMRFs (Table 3). Compared with healthy adolescents with no CMRFs, the ORs (95\% CIs) for pre-diabetes across the clustering of CMRFs (i.e. 1, 2, 3, and 4) were 1.32 (1.03-1.68), 2.07 (1.55-2.76), 2.52 (1.69-3.76), and 5.41 (3.14-9.32), respectively, based on the modified NCEP criteria. The corresponding values were $1.16(0.89-1.51), 1.78(1.35-2.36), 3.07(1.89-4.98)$ and 12.20 (3.93-37.89), respectively, based on the modified IDF criteria (the limited sample size of 18 in the fifth category 


\begin{tabular}{|l|l|l|l|l|l|}
\hline \multicolumn{5}{|l|}{} & \multicolumn{5}{l}{ Clustering of cardio-metabolic risk factors } \\
\cline { 2 - 6 } & $\mathbf{0}$ & $\mathbf{1}$ & $\mathbf{2}$ & $\mathbf{3}$ & $\mathbf{4}$ \\
\hline NCEP criteria & $1.00(\mathrm{ref})$ & $1.35(1.02-1.78)$ & $1.77(1.25-2.52)$ & $1.97(1.28-3.04)$ & $5.22(2.93-9.30)$ \\
\hline IFG & $1.00(\mathrm{ref})$ & $0.95(0.48-1.91)$ & $2.14(0.87-5.25)$ & $4.87(2.06-11.53)$ & $7.61(2.20-26.38)$ \\
\hline IGT & $1.00(\mathrm{ref})$ & $1.61(1.07-2.43)$ & $2.76(1.71-4.44)$ & $3.41(1.85-6.26)$ & $4.67(1.77-12.29)$ \\
\hline Elevated $\mathrm{HbA}_{\mathrm{IC}}$ & $1.00(\mathrm{ref})$ & $1.32(1.03-1.68)$ & $2.07(1.55-2.76)$ & $2.52(1.69-3.76)$ & $5.41(3.14-9.32)$ \\
\hline Pre-diabetes & $1.00(\mathrm{ref})$ & $1.20(0.88-1.64)$ & $1.61(1.14-2.29)$ & $2.28(1.38-3.79)$ & $12.83(4.59-35.82)$ \\
\hline IDF criteria & $1.00(\mathrm{ref})$ & $1.17(0.59-2.32)$ & $1.61(0.65-3.94)$ & $5.67(2.11-15.23)$ & $7.32(1.28-41.99)$ \\
\hline IFG & $1.00(\mathrm{ref})$ & $1.41(0.98-2.04)$ & $2.74(1.77-4.24)$ & $3.32(1.57-7.02)$ & $6.15(1.35-28.11)$ \\
\hline IGT & $1.00(\mathrm{ref})$ & $1.16(0.89-1.51)$ & $1.78(1.35-2.36)$ & $3.07(1.89-4.98)$ & $12.20(3.93-37.89)$ \\
\hline Elevated $\mathrm{HbA}_{\mathrm{IC}}$ & & & &
\end{tabular}

Table 3. Odds ratios $(95 \% \mathrm{CI})$ of pre-diabetes according to clustering of cardio-metabolic risk factors. Adjusted for sex, age, race/ethnicity, and survey years.

of CMRFs resulting in the wider 95\%CI in this category). Similar results were observed for IFG, IGT and elevated HbA1c.

\section{Discussion}

In this pooled analysis of 8 nationally representative population samples of the U.S. adolescents, we found that the risk of pre-diabetes tended to increase with the clustering of CMRFs. Our findings emphasize the need for effective strategies and measures targeting adolescents with clustering of CMRFs to reduce risk of pre-diabetes.

There has been controversy on determining the optimal method to define pre-diabetes in adolescents. IFG, IGT, and elevated $\mathrm{HbAlc}$ have been proposed to have distinct etiological mechanisms ${ }^{18}$ and have poor agreement as indicators of pre-diabetes ${ }^{3,19,20}$. A study using data from NHANES 1999-2014 showed different temporal trends of pre-diabetes as defined by IFG or elevated $\mathrm{HbA1c}{ }^{3}$. Although $\mathrm{HbA1c}$ has the advantages of convenience and less variability during illness, it also has the limitations of lower sensitivity and greater cost, which may affect the number of adolescents classified as patients ${ }^{12}$. Thus, in the present study we investigated the relationship between clustering of CMRFs and risk of pre-diabetes using IFG, IGT and elevated HbAlc, separately, as well as the combination. The results showed that all the indicators were strongly associated with clustering of CMRFs. The assessment of clustering of CMRFs in adolescents may aid pediatricians to identify and treat those with potential risk of pre-diabetes.

We also found that the relationship was not limited to three or more CMRFs. Instead, compared with adolescents with no CMRF, those with two CMRFs also had a significantly higher risk of pre-diabetes. A previous study conducted using NHANES data 2005-2006 $(n=777)$ also demonstrated that adolescents with two or more of the four CMRFs had a higher prevalence of pre-diabetes than those with no $\mathrm{CMRF}^{21}$. Several other studies used subclinical markers of cardiovascular disease as the outcome and showed similar results. A study conducted among 474 adolescents and found that participants who had two or more CMRFs had greater vascular stiffness and wall thickness ${ }^{22}$. Another study using data from the Bogalusa Heart Study $(n=204)$ showed that the atherosclerotic process was accelerated in an exponential manner with the increasing number of CMRFs ${ }^{8}$. Therefore, it is important to note that the risk among adolescents with less than 3 risk factors may be overlooked when using the traditional dichotomous MetS definition, which was referred to the presence of three or more risk factors. A study performed among 461 overweight adolescents aged 10-18 years showed that the best model for diagnosing increased intima-media thickness was the sum of the components of MetS, while the dichotomized variable MetS reduced the diagnostic accuracy ${ }^{23}$. Overall, all these findings suggest that in clinical practice, clinicians should focus attention on children and adolescents with CMRFs clustering instead of a dichotomous definition of MetS. Of note, the greatest increase in the risk of pre-diabetes in this study was seen in adolescents with 4 CMRFs, indicating CMRFs clustering may produce a synergistic effect on pre-diabetes, rather than a simple additive effect ${ }^{24}$.

Our study has several strengths. A major strength is the large, population-based sample size obtained by combining 1999-2014 NHANES data. The large sample size allows us to investigate the patterns of the association across gradients of CMRFs. However, several limitations of our study also warrant consideration. First, the cross-sectional nature of NHANES data precluded the causal inference of CMRFs clustering and adolescent pre-diabetes. Further longitudinal studies are needed to clarify the observed association. Second, the single measurement of PFG, 2-h PG and HbA1c may result in misclassification of pre-diabetes, since glucose measures are subject to variability. However, the ADA does not require a repeat measurement to determine pre-diabetes ${ }^{25}$. Third, there were relatively few cases had 4 CMRFs. However, the results were stable when using different criteria in the study. Fourth, we only included 4 CMRFs in our analysis. Further studies should include other risk factors such as lifestyle factors. Fifth, we treated the four risk factors to have the equal weight in determining pre-diabetes, in accord with the definition of MetS in children. Further studies should validate this assumption. Sixth, the statistical significance is set at $P<0.05$ without consideration of correction for multiple comparisons, which may lead to false positive results. 
In conclusion, this study confirms a positive association between the clustering of CMRFs and pre-diabetes among U.S. adolescents. It might be necessary for effective strategies and measures aiming at adolescents with clustering of CMRF, including those with less than 3 risk factors.

Received: 28 July 2020; Accepted: 1 February 2021

Published online: 03 March 2021

\section{References}

1. Dall, T. M. et al. The economic burden of elevated blood glucose levels in 2012: diagnosed and undiagnosed diabetes, gestational diabetes mellitus, and prediabetes. Diabetes Care 37, 3172-3179 (2014).

2. Fink, B. N. et al. Fruits, vegetables, and micronutrient intake in relation to breast cancer survival. Breast Cancer Res. Treat. 98, 199-208 (2006).

3. Lee, A. M., Fermin, C. R., Filipp, S. L., Gurka, M. J. \& DeBoer, M. D. Examining trends in prediabetes and its relationship with the metabolic syndrome in US adolescents, 1999-2014. Acta Diabetol. 54, 373-381 (2017).

4. Garnett, S. P. et al. Improved insulin sensitivity and body composition, irrespective of macronutrient intake, after a 12 month intervention in adolescents with pre-diabetes; RESIST a randomised control trial. Bmc Pediatr. 14, 289 (2014).

5. Julia, S. et al. Progress and challenges in metabolic syndrome in children and adolescents: a scientific statement from the American Heart Association Atherosclerosis, Hypertension, and Obesity in the Young Committee of the Council on Cardiovascular Disease in the Young; C. Circulation 119, 628-647 (2009).

6. Wilson, P. W., D’Agostino, R. B., Parise, H., Sullivan, L. \& Meigs, J. B. Metabolic syndrome as a precursor of cardiovascular disease and type 2 diabetes mellitus. Circulation 112, 3066-3072 (2005).

7. DeBoer, M. D. \& Gurka, M. J. Low sensitivity of the metabolic syndrome to identify adolescents with impaired glucose tolerance: an analysis of NHANES 1999-2010. Cardiovasc. Diabetol. 13, 83 (2014).

8. Berenson, G.S. et al. Association between multiple cardiovascular risk factors and atherosclerosis in children and young adults. The Bogalusa Heart Study. N. Engl. J. Med. 338,1650-1656 (1998).

9. Magge, S.N., Goodman, E., Armstrong, S.C., COMMITTEE ON NUTRITION; SECTION ON ENDOCRINOLOGY; SECTION ON OBESITY. The Metabolic Syndrome in Children and Adolescents: Shifting the Focus to Cardiometabolic Risk Factor Clustering. Pediatrics 140 (2017).

10. Centers for Disease Control and Prevention. https://www.cdc.gov/nchs/nhanes/index.htm Accessed 20 Nov 2020.

11. Centers for Disease Control and Prevention. Analytic Note Regarding 2007-2010 Survey Design Changes and Combining Data Across other Survey Cycles https://www.cdc.gov/nchs/data/nhanes/analyticnote_2007-2010.pdf Accessed 20 Nov 2020.

12. Classification and Diagnosis of Diabetes. Standards of Medical Care in Diabetes-2018. Diabetes Care 41, S13-s27 (2018).

13. Pavia, M., Pileggi, C., Nobile, C. G. \& Angelillo, I. F. Association between fruit and vegetable consumption and oral cancer: a meta-analysis of observational studies. Am. J. Clin. Nutr. 83, 1126-1134 (2006).

14. Li, B. et al. Intake of vegetables and fruit and risk of esophageal adenocarcinoma: a meta-analysis of observational studies. Eur. J. Nutr. 53, 1511-1521 (2014).

15. Koushik, A. et al. Intake of fruits and vegetables and risk of pancreatic cancer in a pooled analysis of 14 cohort studies. Am. J. Epidemiol. 176, 373-386 (2012).

16. Zimmet, P. et al. The metabolic syndrome in children and adolescents. Lancet 369, 2059-2061 (2007).

17. Cook, S., Weitzman, M., Auinger, P., Nguyen, M. \& Dietz, W. H. Prevalence of a metabolic syndrome phenotype in adolescents: findings from the third National Health and Nutrition Examination Survey, 1988-1994. Arch. Pediatr. Adolesc. Med. 157, 821-827 (2003).

18. Meyer, C. et al. Different mechanisms for impaired fasting glucose and impaired postprandial glucose tolerance in humans. Diabetes Care 29, 1909-1914 (2006).

19. Cowie, C. C. et al. Prevalence of diabetes and high risk for diabetes using A1C criteria in the U.S. population in 1988-2006. Diabetes Care 33, 562-568 (2010).

20. Nowicka, P. et al. Utility of hemoglobin A(1c) for diagnosing prediabetes and diabetes in obese children and adolescents. Diabetes Care 34, 1306-1311 (2011).

21. Li, C., Ford, E. S., Zhao, G. \& Mokdad, A. H. Prevalence of pre-diabetes and its association with clustering of cardiometabolic risk factors and hyperinsulinemia among U.S. adolescents: National Health and Nutrition Examination Survey 2005-2006. Diabetes Care 32, 342-347 (2009).

22. Shah, A. S., Dolan, L. M., Gao, Z., Kimball, T. R. \& Urbina, E. M. Clustering of risk factors: a simple method of detecting cardiovascular disease in youth. Pediatrics 127, e312-318 (2011)

23. Reinehr, T., Wunsch, R., Putter, C. \& Scherag, A. Relationship between carotid intima-media thickness and metabolic syndrome in adolescents. J. Pediatr. 163, 327-332 (2013).

24. Fadini, G. P. et al. A stepwise approach to assess the impact of clustering cardiometabolic risk factors on carotid intima-media thickness: the metabolic syndrome no-more-than-additive. Eur. J. Cardiovasc. Prev. Rehabil. 15, 190-196 (2008).

25. Summary of Revisions. Standards of medical care in diabetes-2017. Diabetes Care 40, S4-S5 (2017).

\section{Acknowledgements}

We thank the National Center for Health Statistic of Centers for Disease Control and Prevention for sharing NHANES data.

\section{Author contributions}

X.P. planned and designed the study. S.W. collated the data. C.L. analyzed data and drafted the manuscript. C.L., S.W. and X.P. contributed to the interpretation of the results and critical revision of the manuscript for important intellectual content and approved the final version of the manuscript. X.P. is the guarantor of this work and, as such, had full access to all the data in the study and takes responsibility for the integrity of the data and the accuracy of the data analysis.

\section{Competing interests}

The authors declare no competing interests.

Additional information

Correspondence and requests for materials should be addressed to X.P. 
Reprints and permissions information is available at www.nature.com/reprints.

Publisher's note Springer Nature remains neutral with regard to jurisdictional claims in published maps and institutional affiliations.

(c) (i) Open Access This article is licensed under a Creative Commons Attribution 4.0 International License, which permits use, sharing, adaptation, distribution and reproduction in any medium or format, as long as you give appropriate credit to the original author(s) and the source, provide a link to the Creative Commons licence, and indicate if changes were made. The images or other third party material in this article are included in the article's Creative Commons licence, unless indicated otherwise in a credit line to the material. If material is not included in the article's Creative Commons licence and your intended use is not permitted by statutory regulation or exceeds the permitted use, you will need to obtain permission directly from the copyright holder. To view a copy of this licence, visit http://creativecommons.org/licenses/by/4.0/.

(C) The Author(s) 2021 